\title{
Effect of Interferential Therapy and Kegel Exercise in Management of Stress Urinary Incontinence-A Comparative Study
}

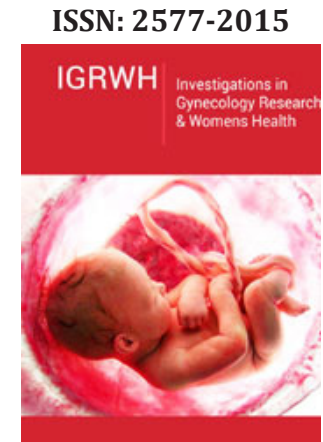

*Corresponding author: Patitapaban Mohanty, Department of Physiotherapy, SVNIRTAR, Cuttack,India

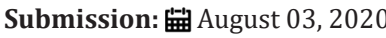

Published: 眥 September 22, 2020

Volume 3 - Issue 4

How to cite this article: Patitapaban Mohanty, Sahoo Radharani, Pattanaik Monalisa. Effect of Interferential Therapy and Kegel Exercise in Management of Stress Urinary Incontinence-A Comparative Study. Invest Gynecol Res Women's Health. 3(4). IGRWH. 000569. 2020

DOI: 10.31031/IGRWH.2020.03.000569

Copyright@Patitapaban Mohanty, This article is distributed under the terms of the Creative Commons Attribution 4.0 International License, which permits unrestricted use and redistribution provided that the original author and source are credited.

\author{
Sahoo Radharani, Mohanty Patitapaban* and Pattanaik Monalisa \\ Department of Physiotherapy, SVNIRTAR, Cuttack,India
}

\section{Abstract}

Introduction: Urinary incontinence is a common problem with widespread human and social implication causing discomfort, shame and loss of self-confidence. It not only affects the quality of life but also has significant cost complications.

Aim of the Study: To find out the effectiveness of kegel exercise, interferential therapy and transverse abdominis strengthening in treating patients having stress urinary incontinence.

Methodology: A total of 50 subjects having stress urinary incontinence at least 6-month duration were recruited randomly. Group 1 experimental group was treated with kegel exercise, interferential therapy and transverse abdominis strengthening and Group 2 conventional group was treated with kegel exercise and transverse abdominis strengthening for five days/week for 4 weeks.

Outcome Measures: Urinary incontinence questionnaire, modified oxford scale and transverse abdominis strength by modified sphygmomanometer. Measurements were taken before and at the end of four weeks of treatment.

Result: The overall results of the study show that there is significant improvement in both the groups. The Group 1 showed significantly better improvement in urinary incontinence questionnaire and modified oxford scale parameters than the Group 2.

Conclusion: Subjects having stress urinary incontinence in between age 20-60 years, because of weak pelvic floor and abdominal muscles was treated with kegel exercise, transverse abdominis strengthening, and interferential therapy was found to be effective.

Keywords: Stress urinary incontinence; Modified oxford scale; Interferential therapy; Transverse abdominis muscle; Pelvic floor muscles

\section{Introduction}

The International Continence Society defined the stress urinary incontinence as the complaint of involuntary leakage of urine during effort or exertion like sneezing or coughing. Stress urinary incontinence is commonly seen after vaginal childbirth that leads to weakness of muscles that support and control the bladder [1]. Urinary incontinence (UI) commonly classified as the stress urinary incontinence (SUI) is characterized by involuntary loss of urine when coughing or sneezing; urge urinary incontinence (UUI) is characterized by an abrupt and sudden urge to urinate that cannot be postponed and mixed urinary incontinence (MUI) is associated with both situation mentioned above [2]. The study of prevalence of urinary incontinence (2013) in Indian women shows that among the total women having incontinence, highest number were found in stress urinary incontinence $(60.8 \%)$ followed by mixed urinary incontinence (26.8\%) and urge incontinence (12.4\%). It was seen that prevalence was low in women up to 30 years of age. In women above 30 years of age, the prevalence ranged from $27.8 \%$ to $42.8 \%$ with maximum prevalence in the age group between 40 to 55 years of age [3].

The prevalence rate of stress urinary incontinence varies between $32 \%$ and $64 \%$. Urinary incontinence affects quality of life and participation in social activities, especially physical activity and exercise [4]. This problem is more pronounced in India, where women usually do not seek treatment for their reproductive health problems and do not vocalize their symptoms. There is a "culture of silence" and low consultation rate among Indian women regarding such problems [5]. Classification of stress urinary incontinence based on severity of its symptoms was defined by Stamey, after Ingelman-Sandberg. Stage I stress urinary incontinence is defined 
as urinary leak during coughing, sneezing and lifting heavy objects. Stage II stress urinary incontinence is diagnosed in patients who perform less exhaustive forms of physical activity, such as walking and switching from sitting to standing. In stage III stress urinary incontinence, urinary incontinence occurs without a physical strain [6].

The main cause of stress urinary incontinence is weakness of pelvic floor muscles supporting the proximal urethra. Hence, the intravesical pressure exceeds the maximal urethral pressure during exertion which results in increased intra-abdominal pressure [7]. Urine leakage occurs when the pressure in the bladder, the expulsive force, is greater than the pressure within the urethra, the closure force. At this point the woman loses urine involuntarily. When there is a weakness in the pelvic floor muscles that support the bladder and other pelvic organs, these organs could prolapse and cause additional pressure on the bladder leading to leakage of urine [8]. De Lancey and Richardson stated that normal pelvic organ support is achieved by support from ligamentous structures above and pelvic floor muscles function from below. Reflex contraction of the pelvic floor with increased intra-abdominal pressure increases urethral pressure more than bladder pressure and maintains continence [9].

Dr. Arnold Kegel, described a pelvic floor exercise, more commonly called a Kegel exercise, consists of repeatedly contracting and relaxing the pelvic floor muscles [7]. Interferential Therapy is a form of medium frequency currents that can be applied to pelvic floor muscles. Interferential Therapy may be used as an adjunct or alone in treatment of urinary incontinence [10]. IC can be applied with two or four electrodes and effective low frequency occurs in that area where the medium frequencies intersect within the pelvis to stimulate the pelvic floor. Ease of usage and external application with- out giving harm to the superficial tissues are the main advantages of this method. The current produces positive responses in the body and the intensity is well tolerated by the patients [11].

\section{Aim of the Study}

To find out the effectiveness of kegel exercise, interferential therapy and transverse abdominis strengthening in treating patients having stress urinary incontinence.

\section{Methodology}

a) Research design: Experimental, two groups, pre-testpost-test structure study design.

b) Research setting: The study was conducted in the Physiotherapy Department of Swami Vivekananda National Institute of Rehabilitation Training and Research (SVNIRTAR).

c) Inclusion criteria: Female subjects having stress urinary incontinence characterized by involuntary loss of urine with coughing, sneezing and lifting weight, duration at least 6 month duration, age $\leq 60$, no cognitive impairment, no neurological deficit. d) Exclusion criteria: Age more than 60yrs, urge and mixed incontinence, neurological deficit, history of hysterectomy, underwent surgery for incontinence, frequent urinary tract infection, COPD.

\section{Outcome measures}

a) Urinary incontinence questionnaire: It is a selfadministered questionnaire to find out the incontinence impact. It has 21 questions. The highest score is 10 . Lowest score is 0 . It includes frequency of urination, nocturnal enuresis, residual urine after urination, activities that leak urine, quantity of urine leaks, protective garments used, sex life affection, confidence level, control over involuntary leak.

b) Modified oxford scale: It is a 6-point grading scale ranges from 0 to 5 used to measure the pelvic floor muscle strength. 0 means no discernible pelvic floor muscle contraction. 5 is a strong pelvic floor muscle contraction. It is the most commonly used scale to measure the pelvic floor muscle strength [12].

c) Modified sphygmomanometer: It is used to measure the core muscle strength. Patient in prone lying position, modified sphygmomanometer was placed horizontally under the abdomen with the lower edge of it just below the ASIS. It was inflated up to $70 \mathrm{mmHg}$ and the patient was instructed to in draw the abdomen. If done properly the pressure drops 6-10 mmHg and patient was asked to maintain up to 10 second. 3 readings were taken, and the mean was taken [13].

\section{Subjects/participants}

A total of 50 female subjects (age range 20-60 years with mean age was 42) having stress urinary incontinence at least 6-month duration were recruited randomly into Group 1 (Experimental group)-25 subjects and group 2 (Conventional group)-25 subjects.

\section{Procedure}

After meeting the inclusion and exclusion criteria through an assessment proforma, informed consent was taken, and subjects were randomly allocated to either of the two groups. All participants underwent an initial baseline assessment of modified oxford scale, urinary incontinence questionnaire and transverse abdominis strength by modified sphygmomanometer. Group-1 (Experimental group) was treated with Interferential therapy, kegel exercise and transverse abdominis strengthening, 5 days per week for 4 weeks. Interferential therapy was given with Vectrostim model, output intensity $0-90 \mathrm{~mA}$, frequency channel I $4000-4250 \mathrm{~Hz}$, channel II $4000 \mathrm{~Hz}$ constant, base $0-100 \mathrm{~Hz}$, Spectrum $0-150 \mathrm{~Hz}$. Patient was positioned in semi-fowlers position. Interferential therapy was given using quadripolar method. Two electrodes were placed on the lower abdomen just above the outer half of the inguinal ligament and another two on the inner aspect of thigh near to the origin of adductor muscle. The parameters used were, intensity up to the tolerable limit of subjects, carrier frequency of $2000 \mathrm{~Hz}$, vector 900 , rhythmic sweep frequency of $10-100 \mathrm{~Hz}$ and duration 20 minutes. At the first treatment session every subject was examined for pelvic floor contraction by finger palpation method using sterilized gloves 
to ensure correct position of the electrodes. Electrodes position was confirmed by checking maximum pelvic floor contraction to a given intensity.

Along with interferential therapy they performed the kegel exercise. The subjects were thoroughly described about the importance and procedure of the exercise. In crook lying position, they were asked to contract the abdomen and in-draw it holds for 5 seconds and then relax for 10 seconds. They were advised to relax the glutei, back muscles and breathe normally. As a progression the contraction time increased up to 10 seconds and rest time decreased up to 5 seconds. Initially they practiced in crook lying position, gradually they were advised to practice it in sitting and standing also. 10 contractions and relaxation forms one set and they were advised to perform 8-10 sets in a day. With abdominal strengthening they were advised to contract the pelvic floor muscles and in-draw that like stopping the mid flow of urine. Contract for 10 seconds and relax for 10 seconds, 10 contraction forms one set, 10 sets of exercise per day for 1 month. Group-2 (Conventional group) was treated with kegel exercise and transverse abdominis strengthening.

\section{Data Collection}

Measurements for all dependent variables were taken prior to the beginning of treatment and at the end 4 weeks of intervention.

\section{Data Analysis}

Statistical analysis was performed using SPSS version 23.0, the dependent variables were analyzed using repeated measures ANOVA. $2 \times 2$ ANOVA. There was one between factor (Group) with two levels (Groups: IFT, kegel exercise and transverse abdominis strengthening) and one within factor (time) with two levels (Pretest, Posttest). In urinary incontinence questionnaire and modified oxford scale pair wise post-hoc comparisons were analyzed using a 0.05 level of significance.

Result

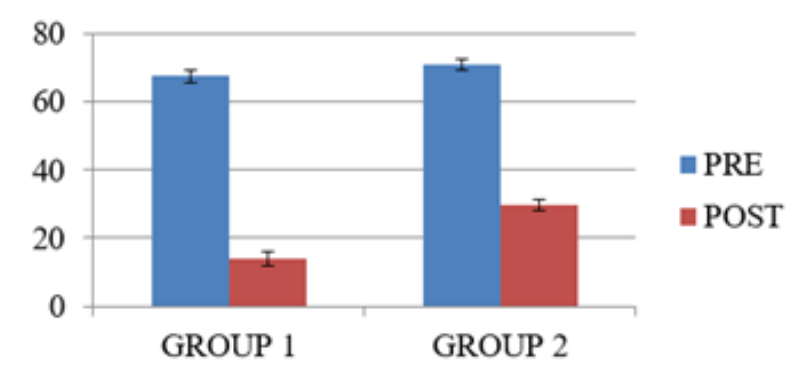

Figure 1: Urinary incontinence questionnaire.

Figure 1 illustrates that there was improvement in quality of life of patients having urinary incontinence measured by urinary incontinence questionnaire in both the groups from pre-treatment to post-treatment. The experimental group showed greater improvement in the post-treatment measurements as compared to the conventional group at the end of 4 weeks of treatment session. There was main effect for time $F(2156.198), d f(1), p=0.000$. There was also a main effect for group $F(17.655)$, df(1), $p=0.000$. The main effects were qualified into time X group interaction $\mathrm{F}$ (38.304), df(1), $p=0.000$. Tukey's Post Hoc analysis shows that there was a significant improvement in quality of life for both the groups. However, the experimental group showed significantly greater improvement than conventional group at the end of 4 weeks.

Figure 2 illustrates that there was improvement in pelvic floor muscle strength measured by modified oxford grading scale in both the groups from pre-treatment to post-treatment. The experimental group showed greater improvement in the post treatment measurements as compared to the conventional group at the end of 4 weeks of treatment session. There was main effect for time $F(1005.231), d f(1), p=0.000$. There was also a main effect for group $F(6.760)$, $d f(1), p=0.012$. The main effects were qualified into time $X$ group interaction $F(8.308), d f(1), p=0.006$. Tukey's Post Hoc analysis shows that there was a significant improvement in pelvic floor muscle strength in both the groups. However, the experimental group showed significantly greater improvement than conventional group at the end of 4 weeks.

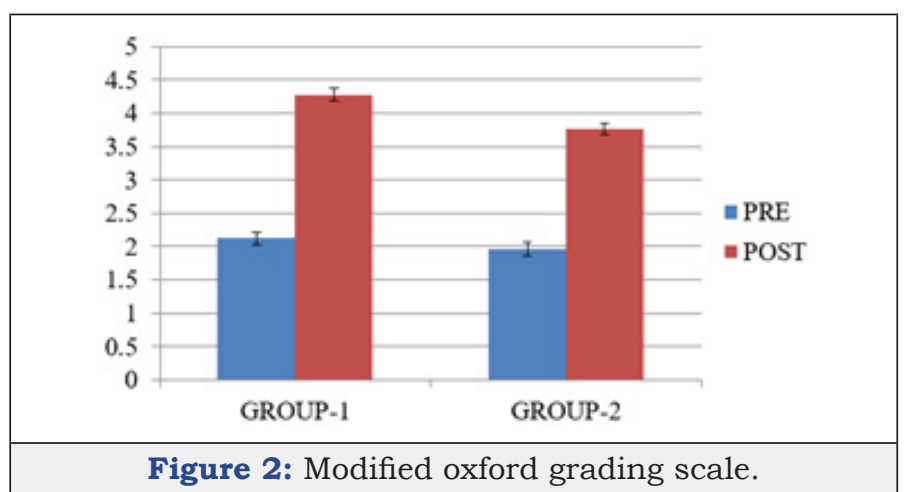

Figure 3 illustrates that there was improvement in transverse abdominis strength measured by modified sphygmomanometer in both the groups from pre-treatment to post-treatment. There was main effect for time $F(959.119), d f(1), p=0.000$. There was also a main effect for group $F(0.853), d f(1), p=0.360$. The main effects were qualified into time X group interaction $F(0.220), \operatorname{df}(1)$, $\mathrm{p}=0.641$.

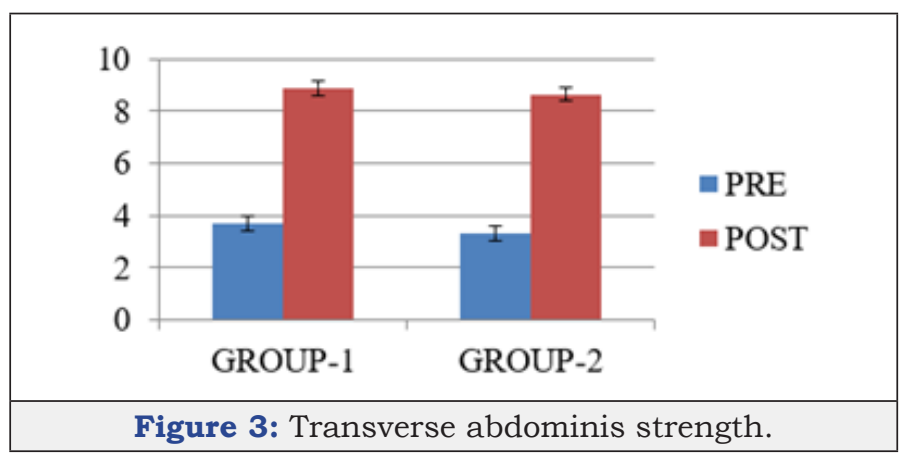

\section{Discussion}

Overall results of the study showed that both the experimental group treated with interferential therapy, kegel exercise, transverse abdominis strengthening and conventional group treated with kegel exercise, transverse abdominis strengthening improved in quality of life measured by urinary incontinence questionnaire, 
pelvic floor muscle strength measured by modified oxford grading scale and transverse abdominis strength measured by modified sphygmomanometer improved from pre-test measurements to post-test measurements. However experimental group improved to a greater extent in urinary incontinence questionnaire and modified oxford grading scale.

\section{Incontinence impact in Urinary incontinence questionnaire}

In this study both the groups showed significant improvement in impact of incontinence, and quality of life, personal and social life over time, but experimental group showed more improvement than conventional group at the end of 4 weeks of intervention. Deep abdominal muscle contraction makes the pelvic floor muscles to cocontract. Co-ordinated co-contraction of pelvic floor muscles with deep abdominal muscles is more effective than specific strength training of the pelvic floor muscles to enhance continence [14]. Bo et al. [15] had conducted a study and found that for stress urinary incontinence; pelvic floor muscle training was more effective than electrical stimulation, vaginal cones, and no treatment control [15]. Cavkaytar et al. [16] conducted a study and found that there was improvement in $68.4 \%$ of the women in the stress urinary incontinence (SUI) and $41.2 \%$ of the women in mixed urinary incontinence (MUI) group, which were statistically significant $(p=0.02)$. Home-based Kegel exercises with no supervision are effective in women with SUI and MUI and the improvement is better in women with SUI [16]. Pelvic floor muscles are responsible for both urethral closure and its pressure increase during a maximal voluntary contraction, supporting the pelvic organs and avoiding their descent during intra- abdominal pressure increase and thus helps in decreasing leakage [17]. During a transverse abdominis muscle maximal voluntary contraction, a pelvic floor muscle cocontraction occurs because of a synergistic activity between the pelvic floor muscle and transverse abdominis muscle that increases the strength and decrease incontinences [18].

Electrical stimulation of pudendal nerve with interferential therapy facilitated contraction of pelvic floor muscles that improved the condition [19]. Interferential therapy which stimulates the efferent fibers of the pudendal nerve at S2 and S3, which innervates the pelvic floor muscles and as direct response at the effector organ the pelvic floor muscles result in increased neural activation causing the pelvic floor muscles to contract [20]. Strengthening weak pelvic floor muscles increases the low urethral closure pressure, thus decrease incontinence improving quality of life. Therefore, pelvic floor re-education methods including Kegel exercises, biofeedback (BF), electrical stimulation, vaginal cones and bladder training can be used in the treatment of stress urinary incontinence [2123]. Electrical stimulation improves the urethral closure pressure by restoration of reflex activation of the pelvic floor muscles, maintaining synchronised contraction of these muscles in addition to the strengthening effect thus decrease incontinence [24].

The reason for improvement in experimental group may be because of improve in strength and endurance of pelvic floor muscles and by giving interferential therapy that restores normal physiological reflexes in the abnormal nerves and muscles. It increases the urethral closure pressure by restoring the reflex activation of the pelvic floor muscles, while encouraging the synchronized contraction of all the pelvic floor muscles increasing their individual strength [25]. Interferential current stimulates pelvic floor muscles in the right sequence when one can't subconsciously or even consciously do so. It can act by inhibiting detrusor muscle contraction [26].

\section{Pelvic floor muscle strength in modified oxford scale}

In this study both the groups showed significant improvement in pelvic floor muscle strength, but experimental group showed more improvement than conventional group at the end of 4 weeks of interventions. In a study of Soni et al. [27] found that there was statistically significant increase in Perineometer power of post Kegel exercise treatment cases in comparison to pretreatment cases as reading in $\mathrm{cm}$ of water of Perineometer was increased from $21.32 \mathrm{cms}$ to $25.08 \mathrm{cms}$. Mean endurance time was increased from 4.97 seconds (pre-treatment) to 7.37 seconds posttreatment cases [27]. Pelvic floor muscles (PFM) tensioning leads to translocation of this muscle layer and internal organs of minor pelvis in cranio-ventral direction, which results in normalization of urethral positioning, an increase in urethral pressure and prevents urine leak [28]. Intensive training of the pelvic floor muscle group results in development of its mass, which provides support for minor pelvis organs and prevents leakage [29]. Morin et al. [30] done Dynamometric studies demonstrated that PFM in women with SUI show lower tone at rest, lower maximum strength, lower contraction speed and lower endurance. With pelvic floor exercises there is increase in strength, contraction speed and increase endurance thus improves the condition [30]. During a cough and the valsalva maneuver, not only women who are incontinent but also nulliparous and parous women who are continent demonstrate bladder neck displacement in the dorso-caudal direction. During voluntary PFM contraction, the position of the bladder neck is elevated in the ventral-cranial direction thus improves better holding and decrease leakage [31-33].

Marques et al. [34] in their study found that there is increase micro-vascularity and oxygenation of pelvic floor muscles with Kegel exercise thus improves the strength and endurance and improve in the condition [34]. After the end of the session it was found that there is also significant improvement in incontinence impact and sexual function measured by urinary incontinence questionnaire. Kegel exercise improves tissue oxygenation and there occurs hypertrophy of pelvic floor muscles which increase contractility, strength and endurance [35]. Soni et al. [27] conducted a study and found that combination of IFT and Kegel exercises increases the strength and endurance in pelvic floor muscles. Increase in endurance was seen by better holding capacity and less or no episode of leakage. The improvement in endurance time was 3.93. They concluded that IFT with Kegel exercise is effective for controlling SUI in the study population [27].

Pelvic floor stimulation enhances the body's natural mechanism and incontinence mechanism by activating appropriate pudendal 
nerve reflexes [36]. IFT produces reflex inhibition of detrusor following contraction of the slow twitch pelvic floor muscles promotes better contraction and improves strength. Interferential therapy stimulates the pudendal nerve and trigger long latency spinal cord reflex response. In addition to direct motor response, this reflex stimulus causes a widespread contraction of pelvic floor muscles. These contractions can strengthen the pelvic floor muscles, elevating the bladder neck and increasing outflow resistance to urine leakage [37]. IFT has an advantage of more deep stimulation and less discomfort is felt by the subject while stimulating the pelvic floor muscles. Slow and fast twitch muscles are activated using the rhythmic sweep frequency [38].

Kai et al. [38] done a study with 24 subjects divided into two groups the experimental group received interferential therapy plus pelvic floor exercises and the control group received pelvic floor exercises only. After 4 weeks of treatment it was found that interferential therapy plus pelvic floor exercise found to be a more effective treatment modality than pelvic floor muscle strengthening exercise alone for incontinence [39].

\section{Transverse abdominis strength in modified sphygmomanometer}

In this study the transverse abdominis strength was measured by the pressure biofeedback unit. It was found that both the groups were improved but there was improvement with time only. The mean improvement in control group is 0.24 and in experimental group is 0.4 respectively. With transverse abdominis strengthening there is hypertrophy of the muscle fibers, increase in strength and have better contraction and coordinated action with the pelvic floor muscles leads to improvement in the stress urinary incontinence [40]. Spasford et al. [41] claimed that deep abdominal muscle contraction makes the pelvic floor muscles to co-contract. Coordinated pelvic floor muscle contraction with deep abdominal muscle contraction is more effective than specific strength training of the pelvic floor muscle to enhance continence [41]. When the patient performs an isometric tension of the abdominal muscles, there occurs activation of Pelvic floor muscles, especially the pubococcygeus muscle and the external anal sphincter [42]. The pelvic floor muscles act in synergism with the muscles of the lumbopelvic region to stabilize the lumbar spine and prevent incontinence.

\section{Conclusion}

Subjects having stress urinary incontinence in between age 2060 years, because of weak pelvic floor and abdominal muscles was treated with kegel exercise, transverse abdominis strengthening and interferential therapy was found to be effective by increasing the strength and endurance of pelvic floor muscles and abdominals as determined by quality of life measured by urinary incontinence questionnaire, pelvic floor muscle strength measured by modified oxford grading scale and transverse abdominis strength measured by modified sphygmomanometer.

\section{Limitations}

Sample size was small, short duration of study, no follow up to see long term effects.

\section{References}

1. Sountoulidis P (2018) Stress urinary incontinence. ICS Committees.

2. Deutchman M, Radcliffe MW (2005) Stress urinary incontinence in women: Diagnosis and medical management. MedGenMed 7(4): 62.

3. Singh U, Agarwal P, Verma ML, Dalela D, Singh N, et al. (2013) Prevalence and risk factors of urinary incontinence in Indian women: A hospitalbased survey. Indian J Urol 29(1): 31-36.

4. Milsom I, Altman D, Lapitan MC, Nelson R, Sillen U, et al. (2009) Epidemiology of urinary (UI) and faecal (FI) incontinence and pelvic organ prolapse (POP). In: Abrams P, Cardozo L, Khoury S, Wein A (Eds.), Incontinence. $4^{\text {th }}$ International Consultation on Incontinence. Committee 1. Health Publication Ltd., France, pp. 35-111.

5. Kumari S, Singh AJ, Jain V (2008) Treatment seeking behavior for urinary incontinence among north Indian women. Indian J Med Sci 62(9): 354356.

6. Ptak M, Brodowska A, Ciećwież S, Rotter I (2017) Quality of life in women with stage 1 stress urinary incontinence after application of conservative treatment-a randomized trial. Int J Environ Res Public Health 14(6): 577.

7. https://shodhganga.inflibnet.ac.in/bitstream/10603/48641/11/11_ chapter\%201.pdf

8. Mahalakshmi L (2013) Effects of physiotherapeutic techniques and combination of physiotherapeutic techniques with core muscle strengthening exercises on stress urinary incontinence and performance in athletic event among collegiate females. International Journal of Science and Research 2(7): 323-329.

9. Brody LT, Hall CM (2007) Therapeutic exercises foundations and concepts. In: Humphrey VN, Colby IA (Ed.), Jaypee Brothers Publication, New Delhi, India, pp. 801-880.

10. Dumoulin C, Seaborne DE, Girardi CQ Sullivan SJ (1996) Pelvic-floor rehabilitation, part 2: pelvic-floor reeducation with interferential currents and exercise in the treatment of genuine stress incontinence in postpartum women-a cohort study. Phys Ther 75(12): 1075-1081.

11. Demirtürk F, Akbayrak T, Karakaya IC, Yüksel I, Kirdi N, et al. (2008) Interferential current versus biofeedback results in urinary stress incontinence. Swiss Med Wkly 138(21-22): 317-321.

12. Ferreira CH, Barbosa PB, Souza FO, Antônio FI, Franco MM, et al. (2011) Inter-rater reliability study of the modified Oxford Grading Scale and the Peritron manometer. Physiotherapy 97(2): 132-138.

13. Souza LAC, Martins JC, Moura JB, Salmela LF, Paula FVR, et al. (2014) Assessment of muscular strength with the modified sphygmomanometer test: what is the best method and source of outcome values? Braz J Phys Ther 18(2): 191-200.

14. Sapsford R (2004) Rehabilitation of pelvic floor muscles utilizing trunk stabilization. Man Ther 9(1): 3-12.

15. Bo K, Talseth T, Holme I (1999) Single blind, randomised controlled trial of the pelvic floor exercises, electrical stimulation, vaginal cones and no treatment in management of genuine stress incontinence in women. $\mathrm{Br}$ Med J 318(7182): 487-493.

16. Cavkaytar S, Kokanali MK, Topcu HO, Aksakal OS, Doğanay M (2015) Effect of home-based Kegel exercises on quality of life in women with stress and mixed urinary incontinence. J Obstet Gynaecol 35(4): 407410. 
17. Miller JA, DeLancey JOL (2007) Functional anatomy of the female pelvic floor. Ann NY Acad 1101: 266-296.

18. Pereira LC, Botelho S, Marques J, Amorim CF, Lanza AH, et al. (2013) Are transversus abdominis/oblique internal and pelvic floor muscles coactivated during pregnancy and postpartum? Neurourol Urodyn 32(5): 416-419.

19. Jiang H, Gill BC, Dissaranan C, Zutshi M, Balog BM, et al. (2012) Effects of acute selective pudendal nerve electrical stimulation after simulated childbirth injury. Am J Physiol Renal Physiol 304(3): F239-F247.

20. Yamanishi T, Kaga K, Fuse M, Shibata C, Uchiyama T (2015) Neuromodulation for the treatment of lower urinary tract symptoms. Low Urin Tract Symptoms 7(3): 121-132.

21. Herman H (1988) Urogenital dysfunction. In: Wilder E (Ed.), Obstetric and Gynecologic Physical Therapy. Churchill Livingstone, New York, USA, pp: 83-113.

22. Ostergard DR, Bent AE (1996) Urogynecology and urodynamics: Theory and Practice. ( $4^{\text {th }}$ edn), Williams and Wilkins, Baltimore, Maryland, USA.

23. Aksac B, Aki S, Karan A, Yalcin O, Isikoglu M, et al. (2002) Biofeedback and pelvic floor exercises for the rehabilitation of urinary stress incontinence. Gynecol Obstet Invest 56(1): 23-27.

24. Turkan A, Inci Y, Fazli D (2005) The short-term effects of physical therapy in different intensities of urodynamic stress incontinence. Gynecol Obstet Invest 59(1): 43-48.

25. Dietz HP, Erdmann M, Shek KL (2012) Reflex contraction of the levator ani in women symptomatic for pelvic floor disorders. Ultrasound Obstet Gynecol 40(2): 215-218.

26. Aarthi M, Sankarganesh A, Sivakumar VPR (2018) Interferential therapy versus pelvic floor exercise for the management of stress urinary incontinence in women. J Physiother Res 2(1): 1.

27. Soni N, Rahule A, Sagdeo V (2014) Evaluation of effect of kegel exercise for the management of stress incontinence in women of Gujrat, India. J Cont Med A Dent 2(3): 20-23.

28. Delancey JOL (1988) Structural aspects of urethrovesical function in the female. Neurourology Urodynamics 7(6): 509-519.

29. Bø K (2004) Pelvic floor muscle training is effective in treatment of female stress urinary incontinence, but how does it work? Int Urogynecol J Pelvic Floor Dysfunct 15(2): 76-84.

30. Morin M, Burbonnais D (2004) Pelvic floor muscle function in continent and stress urinary incontinent women using dynamometric measurements. Neurourol Urodyn 23(7): 668-674.
31. Thompson JA, Sullivan PB, Briffa NK, Neumann P (2007) Comparison of transperineal and transabdominal ultrasound in the assessment of voluntary pelvic floor muscle contractions and functional manoeuvres in continent and incontinent women. Int Urogynecol J Pelvic Floor Dysfunct 18(7): 779-786.

32. Schaer GN, Perucchini D, Munz E, Peschers U, Koechli OR, et al. (1999) Sonographic evaluation of the bladder neck in continent and stressincontinent women. Obstet Gynecol 93(3): 412-416.

33. Marques J, Botelho S, Pereira LC, Lanza AH, Amorim CF, et al. (2013) Pelvic floor muscles training program increases muscular contractility during first pregnancy and postpartum: eletromyographic study. Neurourol Urodyn 32(7): 998-1003.

34. Marques A, Stothers L, Macnab A (2010) The status of pelvic floor muscle training for women. Canadian Urological Association Journal 4(6): 419-424.

35. Shahali S, Kashanian M, Azari A, Salehi R (2010) Effects of pelvic floor muscle exercises on quality of life outcomes in women with stress urinary incontinence. Medical Journal of the Islamic Republic of Iran 24(3): 159-162.

36. Fowler CJ, Griffiths D, Groat WC (2008) The neural control of micturition. Nat Rev Neurosci 9(6): 453-466.

37. Surya GK (2017) Effects of acuteness, Interferential therapy and exercises on the quality of life in women with mixed urinary incontinence-an experimental study. College of Physiotherapy.

38. Kai LS, Naidu J, Cao Y (2003) Additive Effect of Interferential Therapy Over Pelvic Floor Exercise Alone in the Treatment of Female Urinary Stress and Urge Incontinence: A Randomized Controlled Trial. Hong Kong Physiotherapy Journal 21(1): 37-42.

39. Neumann P, Gill V (2002) Pelvic floor and abdominal muscle interaction: EMG activity and intra-abdominal pressure. Int Urogynecol J Pelvic Floor Dysfunct 13(2): 125-132.

40. Sapsford RR, Hodges PW, Richardson CA, Cooper DH, Markwell SJ, et al. (2001) Co-activation of the abdominal and pelvic floor muscles during voluntary exercises. Neurourol Urodyn 20(1): 31-42.

41. Sapsford RR, Hodges PW, Smith MD (2010) Systematic review: Abdominal or pelvic floor muscle training. Neurourol Urodyn 29(5): 800-801.

42. Crommert AEME, Thorstensson A (2009) Trunk muscle reactions to sudden unexpected and expected perturbations in the absence of upright postural demand. Experimental Brain Research 196(3): 385-392. 